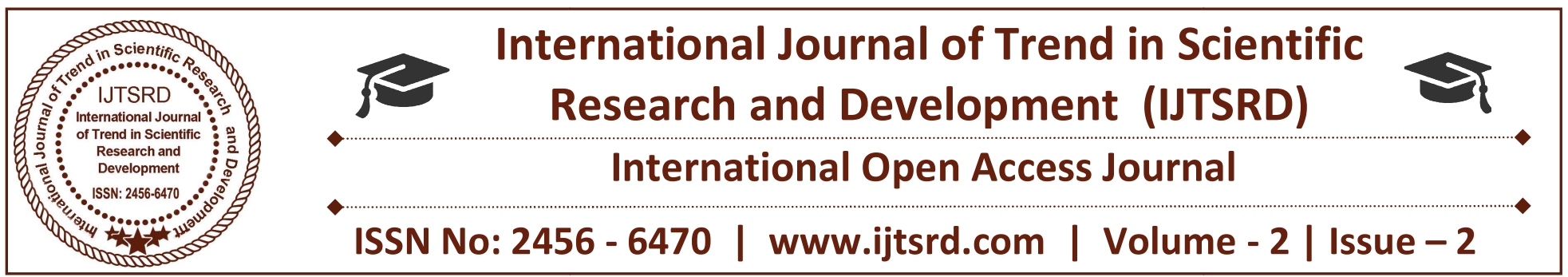

\title{
A Review on Various Plantar Pressure Measurement Systems
}

\author{
Ms. Dhanashri D. Kore \\ M. Tech. Student, Department of Technology, \\ Shivaji University, Kolhapur, \\ Maharashtra, India
}

\author{
Dr. U. L. Bombale \\ Associate Professor, Department of Technology, \\ Shivaji University, Kolhapur, \\ Maharashtra, India
}

\section{ABSTRACT}

Foot plantar pressure is the study of pressure fields acting between the plantar surface of the foot and a supporting surface during everyday locomotion. Such plantar pressure information has proven to be useful in the diagnosis and management of pressure related foot problems, sport mechanics, footwear design, injury prevention and other applications. This paper reviews various foot plantar pressure measurement systems used for different applications. New innovative applications are human identification, biometric, monitoring posture allocation and rehabilitation support system. Gait analysis researchers are focusing on designing systems for uninterrupted measurement of real life parameters which is important in understanding the effect of daily activities on health. The ideal system to achieve this would be mobile, un-tethered, placed in the shoe sole and able to measure plantar pressure effectively in the targeted environment.

Keywords: Foot plantar pressure, pressure sensor, foot problems

\section{INTRODUCTION}

Feet provide the primary surface of interaction with the environment during locomotion therefore it is important to diagnose foot problems at an early stage for injury prevention, risk management and general wellbeing. Examining foot plantar pressure characteristics is one approach to measuring foot health and widely used in various applications. It is, therefore, important that to develop an accurate and reliable foot plantar pressure measurement systems.
The development of miniature, lightweight, and energy efficient circuit solutions for healthcare sensor applications is an increasingly important research focus given the rapid technological advances in healthcare monitoring equipment, microfabrication processes and wireless communication. Typical applications are footwear design, sports performance analysis and injury prevention, improvement in balance control, and diagnosing disease. More recently innovative applications have also been made to human identification, biometric, monitoring posture allocation and rehabilitation support systems.

Foot pressure measurement can be measured in two manners: static and dynamic. Static measurements are taken while the patient is standing and dynamic measurements are taken during locomotion. With static measurement, it is not possible to determine the form or loading pattern of the foot during function. Dynamic measurements are used to determine loading during the actual stance phase of gait, quantified by parameters such as the length of stride, angle of gait and speed of gait, along with many other parameters [11].

The plantar pressure sensing systems commercially available on the market or in research laboratories vary in sensor configuration to meet different application requirements. There are several plantar pressure measurement systems, which can be mainly divided into two categories, i.e., platform systems and in-shoe pressure measurement systems [9]. 
In-shoe foot plantar pressure measurement syetem provide better efficiency, mobility, flexibility and reduced cost measurement systems. For the system to be mobile and wearable for monitoring activities of daily life, the system should be wireless with low power consumption. An on-chip antenna is a practical solution for compact, low cost and for short-range wireless applications because on-chip antenna is feasible with the assistance of rapid scaling of complementary metal-oxide-semiconductor (CMOS) technology.

\section{LITERATURE SURVEY}

Chang-Ming Yang, Chun-Mei Chou, Jwu-Sheng $\mathrm{Hu}$, Shu-Hui Hung, Chang-Hwa Yang [2009] [1] proposed digital textile sensor for measuring gait analysis. Four dome shaped sensors were knitted on each sock. The dome shape sensors were able to record spatio-temporal plantar pressure patterns which were used to calculate the centre of pressure (COP) excursions. Five clip type sensors were sewn to the pant to record lower limb movement. The system was reported to measure the duration of stride cycles and left/right steps, cadence, walking speed, and COP. The microcontroller (Texas Instrument MPS430) and Bluetooth were attached to the wearer"s belt. The digital textile sensors are small and flexible but it is wired to a bluetooth based transmitter device.

Lin Shu, Tao Hua, Yangyong Wang, Qiao Li, David Dagan Feng [2010] [2] developed an in-shoe plantar pressure measurement and analysis system. This system was usedfabric pressure sensing array. They used soft, light weight and high pressure sensitivity textile fabric sensor array. The sensors were connected with a soft polymeric board through conductive yarns and integrated into the insole. A stable data acquisition system was interfaced with the insole. The data was wirelessly transmitted to remote receiver through Bluetooth path. The system was interfaced with desktop, laptop and smart phone and was able to calculate parameters such as mean pressure, peak pressure, centre of pressure (COP) and shift speed of COP. The results were presented for both static and dynamic measurement conditions.

S. Ryan Edgar, Timothy Swyka, George Fulk and Edward S. Sazonov [2010] [3] developed a wearable shoe for rehabilitation of stroke patients. This wearable system is based on the combination of an inshoe sensors and a smartphone, and was designed to operate in free living conditions. The data was collected from a sensor device, used for automatic recognition of postures and activities, characterization of extremity use. And provide behavioral enhancing feedback to patients recovering from a stroke. Edgar et al. claim that the system had $99 \%$ accuracy in the classification.

Thomas Holleczek, Alex Ruegg, Holger Harms, Gerhard Troster [2010] [4] developed "SnowPro", a wearable sport trainer, capable of supporting snowboarders in improving their skill. The system is able to analyze the dynamics of the weight distribution inside the boots. This type of information is essential for identifying the wrong weight shifting techniques, which usually lead to painful crashes in snowboarding sport. The system gives feedback to the user in real-time or after the activity about user performance and support user during learning process. They used three textile integrated pressure sensors, six capacitance-to-digital converters and a Bluetooth module. Besides that, for sport applications the system should not disturb the athlete ${ }^{\text {ee }}$ movement but this system utilized off-the-shelf equipment that is usually very bulky therefore system is not very practical.

Yong Feng, Yunjian Ge, Quanjun Song [2011] [5] proposed dynamic plantar pressure for human identification using an in-sole pressure sensor. They recorded the dynamic pressure data during walking and removed noise of the data using the wavelet transform. They compared the pressure at different positions of key points then identified and classified them using a support vector machine (SVM) running on a PC. The system uses wire to transfer data from the sensor to a data acquisition card on a PC and it is reported that the system has $96 \%$ identification accuracy.

Edward S. Sazonov, James Hill, Yves Schutz, and Raymond Browning [2011] [6] proposed a shoe-based wearable system for monitoring of posture allocations and activities. Monitoring of posture allocations and activities gives accurate estimation of energy expenditure and may aid in obesity prevention and treatment. Many of the existing techniques to recognize postures and activities rely on a multitude of sensors distributed on the body but using multiple sensor locations is the individual burden for everyday use. This paper presented a wearable shoe-based device and related pattern recognition methodology for automatic recognition of six different postures and 
activities. This methodology requires only minimal processing of the sensor data. The shoe sensor was tested in nine adults performing different activities like sitting and standing postures and while walking, running, stair ascent/descent and cycling. Support vector machines (SVMs) were used for classification. This methodology demonstrated excellent recognition accuracy, especially compared to devices that have used only a single location on the body.

Bortolino Saggin, Diego Scaccabarozzi, and Marco Tarabini [2013] [7] described matrological performancse of a plantar pressure measurement system. Plantar pressure measurements provide useful information to diagnose a diverse range of foot disorders; unfortunately, the commercially available measurement systems are undesirably sensitive to several disturbances, but this aspect is mostly neglected in this literature. Results of static and dynamic tests performed on a pedar-X plantar pressure measurement system used for implementation of the sensor model. They reported that the force and center of pressure errors could be reduced by more than $50 \%$ of their initial values.

Carina Gerlach, Dominik Krumm, Marko Illing, Justin Lange, Olfa Kanoun [2015] [8] developed a low-cost, flexible pressure measuring system suitable for everyday use with a simple metrological complexity that can be used to prevent pressure ulcers. To implement a pressure sensor element, a multi-walled carbon nanotube (MWCNT) polydimethylsiloxane (PDMS)-composite was selected. A sensor matrix made of the composite was fully printed to enable pressure distribution measurements. A printed insole was fabricated with six single pressure sensors. These sensors were situated on characteristic points of the insole to detect unhealthy rollover patterns.

Xiaoyou Lin, Boon-Chong Seet [2016] [9] presented a new design of a plantar pressure monitoring system in the form of a smart sock for sensing abnormal changes in the wearer's foot pressures. Authors' group developed a textile-integrated pressure sensor matrix which was knitted inside the sock itself, and RFID technique was applied to collate the sensed readings. With a battery-free design, this system can be powered solely by radio frequency (RF) energy harvested from radio frequency identification (RFID) reader unit hosted on a smartphone of the wearer.
They assembled control unit of the smart sock on a flexible printed circuit board (FPC).

\section{CONCLUSION}

This paper has reviewed major foot plantar pressure measurement systems available in the market and reported in the current literature. In designing of plantar pressure measurement systems, the main requirements are accuracy, sensitivity, spatial resolution, sampling frequency and calibration. It is important that the system should be mobile and wearable for monitoring activities of daily life, for that the system should be wireless with low power consumption also. Wireless in-shoe foot plantar measurement systems have potential application to better data transfer communication systems, small sized biomedical sensors and other uses.

\section{REFERENCES}

1. Chang-Ming Yang, Chun-Mei Chou, "A wireless gait analysis system by digital textile sensors," in Annual International Conference of the IEEE EMBS Minneapolis, Minnesota, USA, September 2-6, 2009.

2. Lin Shu, Tao Hua, Yangyong Wang, Qiao Li, David Dagan Feng, "In-Shoe Plantar Pressure Measurement and Analysis System Based on Fabric Pressure Sensing Array," IEEE Transactions on Information Technology in Biomedicine, Vol. 14, NO. 3, May 2010.

3. S. Ryan Edgar, Timothy Swyka, George Fulk and Edward S. Sazonov, "Wearable Shoe-Based Device for Rehabilitation of Stroke Patients," Annual International Conference of the IEEE EMBS Buenos Aires, Argentina, pp. 3772-3775, 31 August-4 September 2010.

4. Thomas Holleczek, Alex Ruegg, Holger Harms, Gerhard Troster, "Textile Pressure Sensors for Sports Applications" IEEE Sensors 2010 Conference, November 2010.

5. Yong Feng, Yunjian Ge, Quanjun Song, "A Human Identification Method Based on Dynamic Plantar Pressure Distribution," Proceeding of the IEEE International Conference on Information and Automation Shenzhen, China, pp. 329-332, June 2011.

6. Edward S. Sazonov, James Hill, Yves Schutz, and Raymond Browning, "Monitoring of Posture Allocations and Activities by a Shoe-Based 
Wearable Sensor," IEEE Transactions on Biomedical Engineering, Vol. 58, No. 4, April 2011.

7. Bortolino Saggin, Diego Scaccabarozzi, and Marco Tarabini, "Metrological Performances of a Plantar Pressure Measurement System," IEEE Transactions on Instrumentation and Measurement, Vol. 62, No. 4, April 2013.

8. Carina Gerlach, Dominik Krumm, Marko Illing, Justin Lange, Olfa Kanoun, "Printed MWCNTPDMS-Composite Pressure Sensor System for Plantar Pressure Monitoring in Ulcer Prevention," IEEE Sensors Journal, 2015.

9. Xiaoyou Lin, Boon-Chong Seet, "Battery-Free Smart Sock for Abnormal Relative Plantar Pressure Monitoring," IEEE Transactions on Biomedical Circuits and Systems, 2016.

10. Gefen, "A. Pressure-sensing devices for assessment of soft tissue loading under bony prominences: Technological concepts and clinical utilization," Wounds 2007, 19, 350-362.

11. Thanh Dinh, Aristidis Veves, Francesco Tecilazich, "Measuring Pressure in the Diabetic Foot," Measurements in Wound Healing, pp. 17519, May 2012. 